\title{
Research on Routing Optimization Problem of Cigarette Logistics Vehicle with Time Window Based on Hybrid Genetic Algorithm
}

\author{
Wenbin Fan ${ }^{1, a^{*}}$ and Wen Feng ${ }^{2, b}$ \\ 'Zhengzhou University, Zhengzhou, Henan, China \\ ${ }^{2}$ Zhengzhou University, Zhengzhou, Henan, China \\ aiewbfan@zzu.edu.cn, biefengwen@163.com
}

\begin{abstract}
Keywords: Cigarette distribution; Hybrid genetic algorithm; Routing optimization; Logistics distribution; Matlab
\end{abstract}

\begin{abstract}
Vehicle Routing Problem is key step in logistics distribution; excellent distribution strategy helps enterprises in improving service experience and reducing service cost. In logistics distribution system for cigarette, huge demand point amount will always be faced with, and customers will put forward requirements for time quantum of accepting service; this Paper researches cigarette distribution routing optimization problem within this big range. By combining actual demands, establish mathematical model for cigarette logistics routing optimization, and use genetic algorithm to solve this problem; propose genetic algorithm-based hybrid genetic algorithm using hill climbing algorithm for local optimization; carry out Matlab simulating calculation through algorithm case; compared to common genetic algorithms, new hybrid algorithm can increase algorithm convergence speed and also improve globally optimal solution quality of algorithms.
\end{abstract}

\section{Introduction}

China is the top tobacco country in the world; both annual cultivated area for tobacco and production and sale quantity of tobacco account for one third of the world amount, in which the output of flue-cured tobacco and cigarette is the first in the world. According to state tax component study, tobacco industry tax can cover $8 \%-10 \%$ of total state tax; therefore, tobacco industry is also a key industry in national economy. Tobacco tax in some economically undeveloped areas in our country is even called "tobacco finance". Improving management level in tobacco industry and reducing operating cost for tobacco in our country are of vital strategic importance in increase of our national economy and fiscal revenue. For distribution arrangement, there are always various distribution schemes to be selected by logistics enterprises; however, different distribution schemes have different transportation costs; therefore, for enterprises, on the precondition of meeting constraint conditions, to select a distribution strategy which is most economical and effective has become one important means to reduce enterprise operating cost.

In cigarette distribution logistics area, logistics distribution routing optimization is actually a problem of NP; for this kind of problems, especially when distribution points are in large number, traditional algorithm can be used, but solving result will not be satisfying. Genetic algorithm, as a modern heuristic intelligent bionic algorithm, is a relatively effective global searching algorithm which has characteristics of simple operation and quick convergence speed; but relatively, genetic algorithm has no outstanding capacity in local optimization. For this reason, this Paper tries to combine it with hill climbing algorithm which has relatively strong local searching capacity to make up deficiency of genetic algorithm in this aspect; example verification and performance analysis given in the end of the Thesis are used to prove the feasibility of hill climbing genetic algorithm.

\section{Establishment of Mathematical Model for Cigarette Logistics Distribution Routing Optimization}

During cigarette logistics distribution, there will usually be a distribution center and several demand customer points; the number of vehicles used in cigarette distribution, routes passed 
through, distribution routing reasonability, and time window for customer to accept service have direct influence on distribution efficiency and total distribution cost; therefore, adopting intelligent and reasonable method to determine distribution strategy so as to form an excellent vehicle routing planning algorithm is a vital working step in cigarette logistics management. Objectives of vehicle distribution routing optimization can be various; considering actual conditions of cigarette distribution, in this Paper, it is supposed that there is only one distribution center (but not limited in amount); distribution vehicles for using are of same specification; goods are delivered to multiple demand points having time requirements; each distribution point has coordinate, distribution volume, and time interval for accepting service; and in case distribution is not carried out within demand point interval, there will be certain multiplication cost; it is required to formulate reasonable and efficient distribution strategy so as to realize the lowest total distribution cost and the highest customer satisfaction. Based on the above demands, we determine optimization objectives of distribution routing strategy considering the following: (1) optimize vehicle distribution strategy so as to make the cost of this strategy be the lowest; (2) deliver goods according to demand time window of demand points so as to improve customer experience; (3) under the precondition of meeting demands of demand points, one demand point shall has one and only one distribution vehicle to complete.

According to the above, cigarette logistics distribution routing to be optimized in this Paper can be expressed as follows: enterprise owns $K$ distribution vehicles; each vehicle has maximum load of $Q$; there are $n$ customer demand points $(1,2, \ldots, n)$ which have different demands for cigarette at present; quantity demanded by the number $i$ customer demand point is $d_{i}(i=1,2, \ldots, n)$ and there is $\max d_{i} \leq Q$; namely, demand of each point can be completed by one vehicle; the time for vehicle to reach customer $i$ is $s_{i}$; the time needed to meet demand of customer $i$ is $T_{i}$; time interval for accepting service of demand point is $\left[e_{i}, l_{i}\right]$; namely, in case the time for distribution vehicle to reach customer $i$ is less than $e_{i}$, the vehicle will wait at point $i$ and thus generate waiting cost; in case it is more than $l_{i}$, there will be delay penalty cost; suppose distribution vehicle cost for unit transport distance is $\alpha$, fixed start using cost of unit vehicle is $\beta$; set the time for vehicle to move from customer $i$ to customer $j$ and $i \neq j$; transport mission from each customer can and shall be completed by one distribution vehicle at a time; each vehicle departs from the distribution center and returns back to the distribution center finally. The final objective is to find out distribution strategy for cigarette with the lowest cost under the following constraint conditions. According to the above suppositions, the following model can be got:

$$
\begin{aligned}
& \min F(i, j, k)=\alpha \sum_{k=1}^{K} \sum_{j=0}^{N} \sum_{i=0}^{N} c_{i j} x_{i j k}+\beta \sum_{k=1}^{K} \sum_{j=1}^{N} x_{0 j k}+\sum_{k=1}^{K} \sum_{j=1}^{N} P_{i}\left(s_{i}\right) \\
& \text { s.t } \sum_{\mathrm{k}=1}^{\mathrm{K}} \sum_{j=1}^{\mathrm{N}} x_{i j k} \leq K \quad i=0 \\
& \sum_{\mathrm{j}=1}^{\mathrm{N}} x_{i j k}=\sum_{j=1}^{N} x_{i j k} \leq 1 \quad i=0, k \in\{1,2, \ldots, K\} \\
& \sum_{k=1}^{K} \sum_{j=0}^{N} x_{\mathrm{ijk}}=1 \\
& \sum_{\mathrm{k}=1}^{\mathrm{N}} \sum_{\mathrm{i}=0}^{\mathrm{N}} x_{\mathrm{ijk}}=1 \\
& \quad j \in\{1,2, \ldots, N\}
\end{aligned}
$$




$$
\begin{aligned}
& \sum_{i=0}^{\mathrm{N}} \sum_{j=0}^{\mathrm{N}} d_{i} x_{i j k} \leq Q \quad k \in\{1,2, \ldots, K\} \\
& \sum_{i=0}^{N} x_{i p}-\sum_{j=0}^{N} x_{p j}=0 \quad p \in\{1,2, \ldots, N\}, k \in\{1,2, \ldots K\} \\
& T_{0}^{k}+\sum_{i=0}^{N} \sum_{j=0}^{N} x_{i j k}\left(t_{i j}+f_{i}+w_{i}\right) \leq T_{R}^{k} \quad k \in\{1,2, \ldots, K\} \\
& \sum_{k=1}^{K} \sum_{i=0}^{N} x_{i j k}\left(s_{i}+f_{i}+w_{i}+t_{i j}\right)=s_{j} \\
& x_{i j k} \in\{0,1\} \quad j \in\{1,2, \ldots, K\} \\
& P_{i}\left(s_{i}\right)=p \max \left(e_{i}-s_{i}, 0\right)+q \max \left(s_{i}-l_{i}, 0\right) \\
& P_{i}\left(s_{i}\right)=\left\{\begin{array}{cc}
p\left(e_{i}-s_{i}\right) & s_{i}<e_{i} \\
0 \quad & e_{i} \leq s_{i} \leq l_{i} \\
q\left(s_{i}-l_{i}\right) \quad & s_{i}>l_{i}
\end{array}\right.
\end{aligned}
$$

Eq. 1 indicates total cost of optimized routing which is composed of vehicle travel distance cost, cost of vehicle using number, and penalty cost for service of non-service window these three kinds; Eq. 2 indicates upper number limit $K$ of transport machine which can execute distribution mission; Eq. 3 indicates that both initial point and terminal point of each vehicle are distribution center; Eq. 4 and Eq.5 indicate that demand point of each customer can only be served by same distribution vehicle for once; equation Eq. 6 indicates that the upper load limit of each vehicle can meet total demand of mission needs to be completed by the vehicle; equation Eq.7 indicates that vehicle reaches service point and leaves that point after completing service; equation Eq.8 indicates that distribution mission can be finished within specified working time of the center; equation Eq.10 refers to integer constraint which restricts that $x_{i j k}$ can only be taken as 0 or 1; Eq.11 is a expression of penalty cost formed by integrating equation Eq.12. The above model is a more general problem including TSP and VRP problems. Objective function and constraint conditions considered in this Paper are relatively comprehensive and close to practical problems; the got solution is also a global solution to practical problems and it can provide good reference to the solution of practical problems.

\section{Algorithm Overview}

Genetic Algorithm Overview. Genetic algorithm is a calculation model related to biological evolution process which simulates natural selection in Darwin's biological evolutionism and genetics mechanism; it is a genetic and evolutionary process which simulates biont's survival of the fittest and survival of the fittest in natural environment, and it is also an algorithm which searches for optimal solution through natural evolution process simulation. In 1967, Bagley J.D proposed the concept of genetic algorithm; Professor J.H Olland in American Michigan University had firstly carried out systematic research on genetic algorithm in 1975; genetic algorithm is an algorithm which searches for global optimization based on rule of survival of the fittest; in the algorithm, chromosome which possesses the best viability will have the biggest possibility to survive. The common point between optimization problem and biological evolution rules makes it possible to obtain optimal solution to optimization problem by using genetic algorithm. In order to improve genetic algorithm, this Paper will place hill climbing algorithm with relatively good local optimization capacity into genetic algorithm and take it as an independent operator so as to prevent genetic algorithm being sunk into local solution and to increase convergence speed and accuracy of 
genetic algorithm.

Hill Climbing Algorithm Overview. Hill climbing algorithm is a search algorithm which has good local optimization effect and relatively weak global optimization effect; firstly, randomly select one point in search space as initial point of iteration; then randomly search a point in its neighborhood and calculated its function value; in case the function value of this point is better than that of initial point, continue searching by taking this point as initial point; if not, search another point and compare it with initial point; in case no better point is searched for several times, terminate the search. During handling of local optimization problem, hill climbing algorithm can rapidly converge to optimal point; however, there are multiple local optimal points in global optimization, and hill climbing algorithm can only find out one of these local optimal points; although hill climbing method can not be used in local optimization, it can carry out optimization through randomly searching individual in neighborhood without using gradient; therefore, in handling complex problems through genetic algorithm, hill climbing algorithm can assist genetic algorithm in local optimization thus to improve convergence and accuracy of the algorithm.

\section{Algorithm Implementation Operation}

Chromosome Coding. Coding and decoding mode to genetic algorithm is just like foundation to tall buildings; to select appropriate coding and decoding mode is the foundation of algorithm. According to different demands of practical problems, researchers of previous generations had also proposed different coding and decoding strategies which can be divided into real number coding, binary coding, integer coding or alphabetical coding, and data structure coding related to specific problems. Cigarette distribution routing optimization problem to be solved in the Thesis is a problem of combinational optimization; adopt natural number coding mode based on demand point for chromosomes. Suppose 3 vehicles are used to distribute goods for 9 demand points and we get individual coding (0 325079640180 ), among which 0 refers to distribution center; then routing scheme represented by this chromosome is: subrouting 1: (0 - 3 - 2 - 5 - 0); subrouting 2: (0 - 7 - 9 - 6 -4); subrouting 3: (0 - $1-8-0)$. Use distribution center 0 as separator for different subroutings to make each chromosome directly see routing distribution clearly; however, this coding mode will bring a large number of useless solutions to following interlace operation. In order to avoid this, coding strategy adopted in the Thesis is to not indicate partition section in chromosomes and successively arrange chromosomes in all routings; in this case, coding mode for the above chromosomes in the Thesis is (3 25796418 ), which can greatly simplify the handling for constraint conditions of the model.

Fitness Function. The natural law of "survival of the fittest, survival of the fittest" is the key rule in biological evolution process in natural world; biont's adaptive capacity to environment is the basic standard for measuring quality of this species. So similarly, fitness is the standard for judging whether individual quality is good in genetic algorithm; individual which has higher fitness is a better one. The Thesis is to minimize combinational optimization problem, and the objective is to realize minimum total distribution cost, namely minimum objective function value; therefore, we transform objective function into fitness through the following methods:

$$
f_{i}=\frac{1}{z_{i}} \quad i \in\{1,2, \ldots, \text { popsize }\}
$$


$z_{i}$ in Eq.13 refers to the solution of scheme related to the number $i$ chromosome in population and the distribution cost for scheme of the number $i$ chromosome. $f_{i}$ refers to related fitness value of the number $i$ chromosome; the bigger it is, the probability for the next generation to possess its characteristics will be higher.

Crossover Operator. In contemporary natural science, the essence of biological evolution and inheritance is actually the process of mutual confluence and crossing of two chromosomes which have their own characteristic genes; in genetic algorithm, crossover operator of great importance is adopted to reproduce crossing and recombination in evolutionary process. On the one hand, it retains characteristics of excellent individual in original group; on the other hand, it promotes the appearance of new individual and maintains individual diversity of new-generation population at the same time. Method adopted in the Thesis is Partially Matched Exchange Method (PMX for short). PMX Method is different from traditional crossover operator for it is not to directly exchange crossing section of original chromosomes; besides, before taking out same genes in original chromosomes one by one, crossing section is placed before the first gene of original chromosomes. Suppose there are 9 demand points in total, two chromosomes $A(123456789)$ and $B(987654321)$; then randomly generate two crossing sections and replace genes at the middle of crossing sections and place them at the front of the first gene of these two original chromosomes; finally, take out same genes in original chromosomes and obtain new chromosome; for example,

$$
\begin{aligned}
& A:(123456789) \quad B:(987654321) \\
& \Downarrow \text { Select crossing section } \Downarrow \\
& A^{\prime}:(123|4567| 89) \quad B^{\prime}:(987|6543| 21) \\
& \Downarrow \text { Cross to remove replacemen } \mathrm{t} \Downarrow \\
& A^{\prime \prime}:(6543 \mid 123456789) \quad B^{\prime \prime}:(4567 \mid 987654321) \\
& \Downarrow \text { Delete repeated genes } \Downarrow \\
& A_{1}:(654312789) \quad B_{1}:(456798321)
\end{aligned}
$$

take No.3 point and No.7 point as crossing points and then get $A^{\prime}(123|4567| 89)$ and $B^{\prime}(987|6543| 21)$; carry out replacement operation to get $A^{\prime \prime}(6543 \mid 123456789)$ and $B^{\prime \prime}(4567 \mid 987654321)$; take out repeated genes to obtain new individuals $A_{1}(654312789)$ and $B_{1}(456798321)$. Compared with traditional single-point crossover and multi-point crossover, PMX can guarantee that new individuals can be obtained even after crossing two same individuals, thus avoiding prematurity phenomenon and reducing the probability of result being local optimal solution.

Mutation Operator. Evolution of species can not be separated from mutation, which guarantees the continuousness of species diversity; mutation operator is brought into algorithm to reflect species mutation phenomenon and its probability is generally set as [0-0.02]. The possibility of species mutation is small; therefore, the main function of mutation operator in genetic algorithm is to assist evolution; besides, it also plays an important role in maintaining population diversity and avoiding prematurity, thus improving the performance of searching global solution in genetic manipulation. In this Paper, random reverse order mode is adopted to generate mutation; for example:

$$
A(123456789) \Rightarrow A^{\prime}(123|45678| 9) \Rightarrow A_{1}(123876549)
$$


Evolution Termination Rule. Genetic algorithm belongs to random search algorithm; therefore, at the time of executing algorithm, it is required to preset evolution termination rule for terminating evolution circulation of genetic algorithm; when algorithm meets preset termination rule, stop iteration; to preset evolution algebra can reliably control operation time and solution accuracy of genetic algorithm; therefore, preset for evolution algebra is adopted as termination rule in this Paper; when iteration time reaches preset value, terminate algorithm circulation and derive optimal solution; otherwise, algorithm circulation will continue and circulation time will accumulate.

Algorithm Steps. Step1: use algorithm case data to generate first-generation population of distribution routing randomly; Step2: set algorithm parameters and termination conditions such as $\alpha 、 \beta 、 p 、 q 、 P c 、 P m 、 G e n$; Setp3: mark evolution algebra as 0 , and generate $N$ chromosomes randomly; improve the next generation according to roulette wheel method based on ordination and preservation strategy for optimal individual; Step4: calculate fitness of each chromosome, use PMX crossover operator to complete crossing step, use mutation operator to carry out random mutation, recombine new population, carry out hill climbing operation, mark iteration time as +1 ; Step5: judge whether price adjustment termination condition is met; if so, output distribution routing, total cost, and iteration time; if not, skip to Step4.

\section{Hill Climbing Operation In Hybrid Genetic Algorithm}

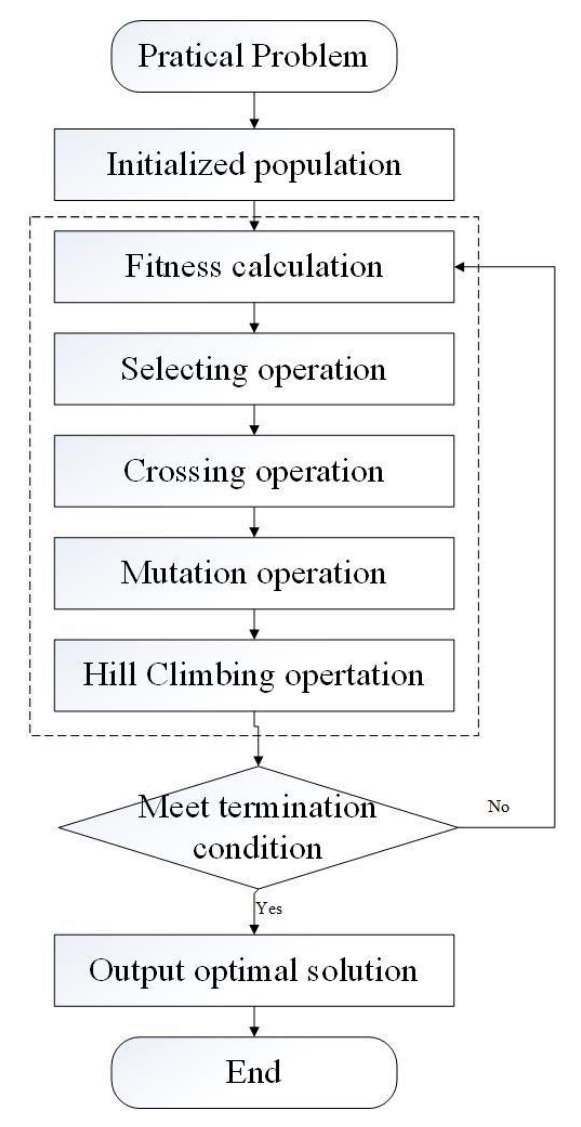

Figure 1. Flow chart of hybrid genetic algorithm

There will be optimal individuals in new-generation population got from the algorithm; carry out hill climbing operation for them and it can effectively prevent genetic algorithm being sunk into local optimal solution and also improve convergence effect of the algorithm. In order to use implementation of hybrid algorithm of genetic algorithm and hill climbing algorithm, in this Paper, it is proposed to place hill climbing algorithm which has relatively better local optimal solution 
solving capacity into locally optimal solution and to take it as an independent step; adopt gene exchange method to carry out hill climbing operation and the steps are: take the optimal individual in new-generation optimal as optimal; exchange any two genes of it; in case exchange result is better than optimal individual, replace that optimal individual; repeat hill climbing operation until reaching certain exchange time.

\section{Simulation Verification and Performance Analysis}

Experimental Data. Operation environment for solving problem in this Paper is as follows: processor is Intel Core i5-4590; memory is 4G; operating system is Windos 10; Matlab operating version is R2015b.

It is supposed that there are 100 demand points which require distribution in this Paper; distribution data includes parameters of coordinate, demand quantity, the earliest time for accepting service, the latest time for accepting service, and service time of each demand point; please refer to Table 1. The coordinate of distribution center is $(40,50)$. Please refer to algorithm case $\mathrm{C} 107$ in Benchmark Problems designed by Solomon in 1983 for detailed experimental data.

Table 1 Distribution Customer Coordinate

\begin{tabular}{ccccccc}
\hline $\begin{array}{c}\text { CUST } \\
\text { NO. }\end{array}$ & $\begin{array}{c}\text { XCPPR } \\
\text { D. }\end{array}$ & $\begin{array}{c}\text { YCOORD } \\
\text {. }\end{array}$ & DEMAND & $\begin{array}{c}\text { READY } \\
\text { TIME }\end{array}$ & DUE DATE & $\begin{array}{c}\text { SERVICE } \\
\text { TIME }\end{array}$ \\
\hline 0 & 40 & 50 & 0 & 0 & 1236 & 0 \\
1 & 45 & 68 & 10 & 850 & 1030 & 90 \\
2 & 45 & 70 & 30 & 758 & 938 & 90 \\
3 & 42 & 66 & 10 & 16 & 196 & 90 \\
4 & 52 & 68 & 10 & 665 & 845 & 90 \\
5 & 42 & 65 & 10 & 15 & 195 & 90 \\
$\ldots$ & $\ldots$ & $\ldots$ & $\ldots$ & $\ldots$ & $\ldots$ & $\ldots$ \\
99 & 55 & 80 & 10 & 695 & 872 & 90 \\
100 & 55 & 85 & 20 & 597 & 777 & 90 \\
\hline
\end{tabular}

Line I in Table 1 is the serial number of cigarette distribution center and customer demand points; line II and III respectively represent coordinate $x$ and coordinate $y$ of related point; values in line IV represent cigarette amount required by customer demand points; line V and VI show time interval for accepting service of customer demand points; line VII indicates the time needed to serve related demand point. Table 1 shows location distribution of each point in experimental data, in which red circle represents cigarette distribution center. 


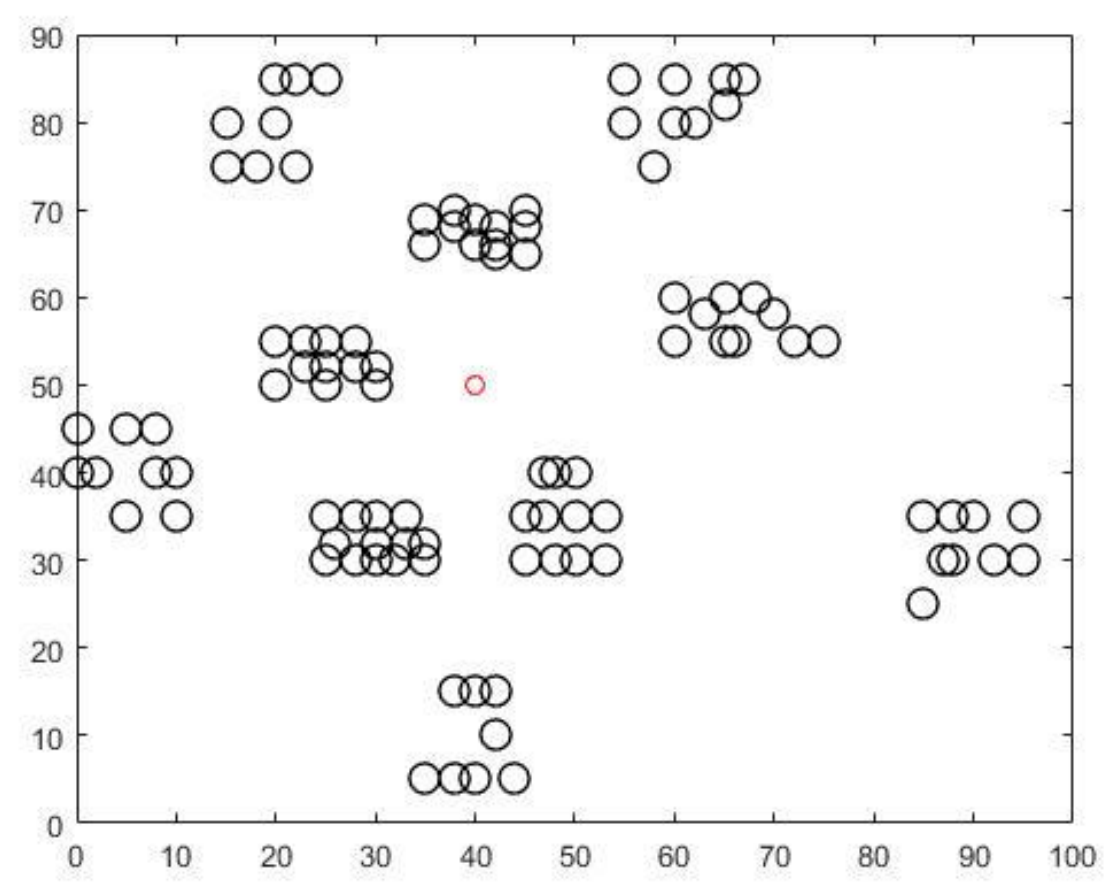

Figure 2. Distribution Diagram of Point Position in Experiment Algorithm Case

Experimental Parameter Setting. In this Paper, algorithm case C107 is used as experimental data for solving problem in the Thesis. During solution solving, related experiment parameters are set; see Table 2:

\begin{tabular}{|c|c|c|c|}
\hline No. & Parameter & Meaning & Experiment value \\
\hline 1 & $\alpha$ & $\begin{array}{l}\text { Operation cost for unit } \\
\text { distance }\end{array}$ & 5 \\
\hline 2 & $\beta$ & Start using cost for unit vehicle & 100 \\
\hline 3 & $p$ & Waiting cost for unit time & 1 \\
\hline 4 & $q$ & Delay cost for unit time & 1.5 \\
\hline 5 & $Q$ & Unit vehicle capacity & 200 \\
\hline 6 & popsize & Population size & 200 \\
\hline 7 & Gen & Evolution termination algebra & 1000 \\
\hline 8 & pcrossover & Crossover probability & 0.85 \\
\hline 9 & pmutation & Mutation probability & 0.02 \\
\hline
\end{tabular}

Hybrid Hill Climbing and Genetic Algorithm Comparison. After placing hill climbing operation into original algorithm, use respective algorithm case C107 of original scheme and new scheme to solve the problem for 10 times at a time, and the optimal strategy for these two strategies will be got as follows: 


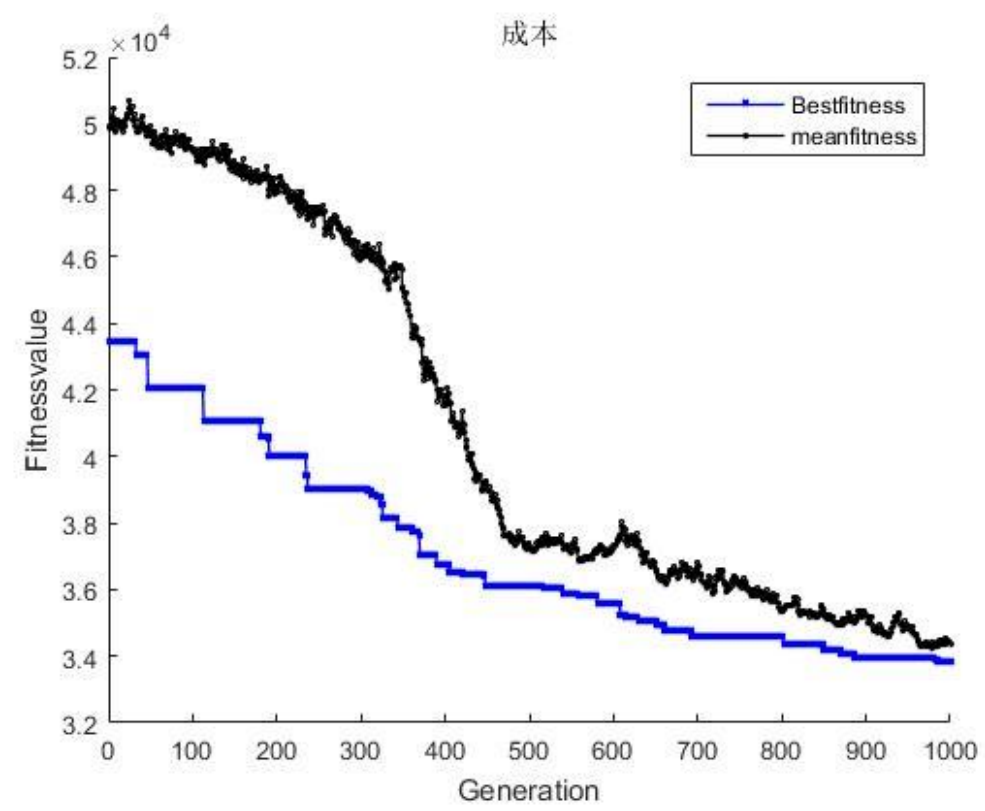

Figure 3. Evolution Graph of General Genetic Algorithm

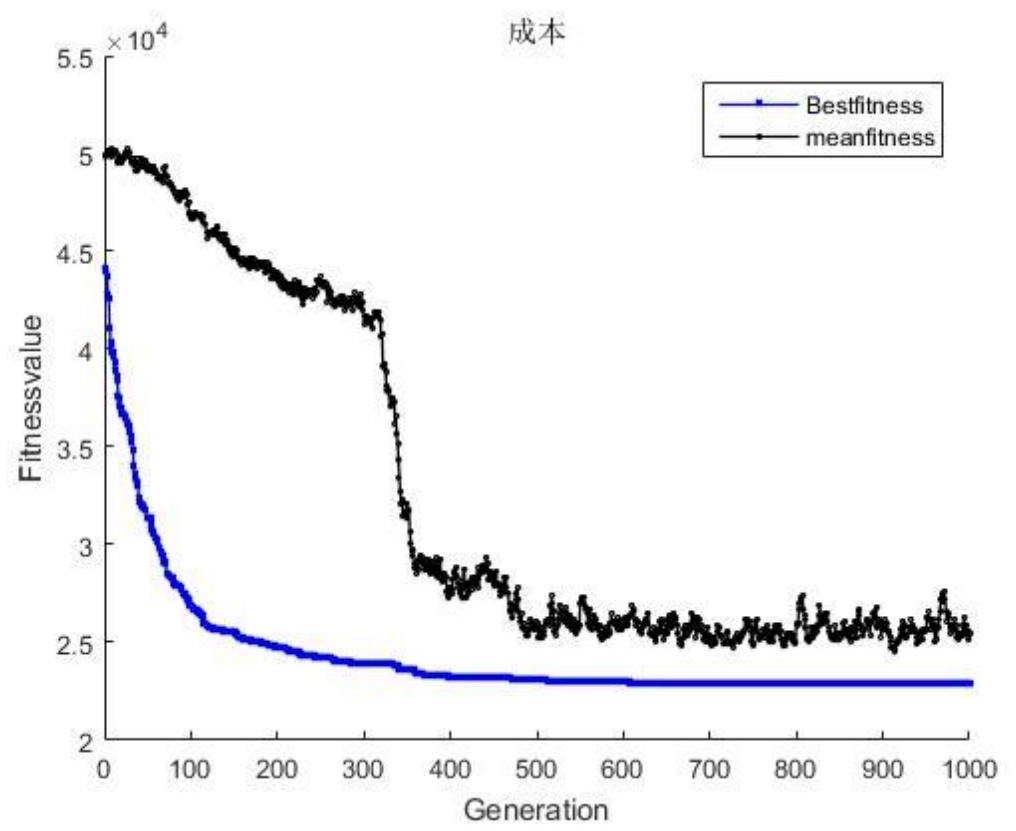

Figure 4. Evolution Graph after Adding in Hill Climbing Operation 


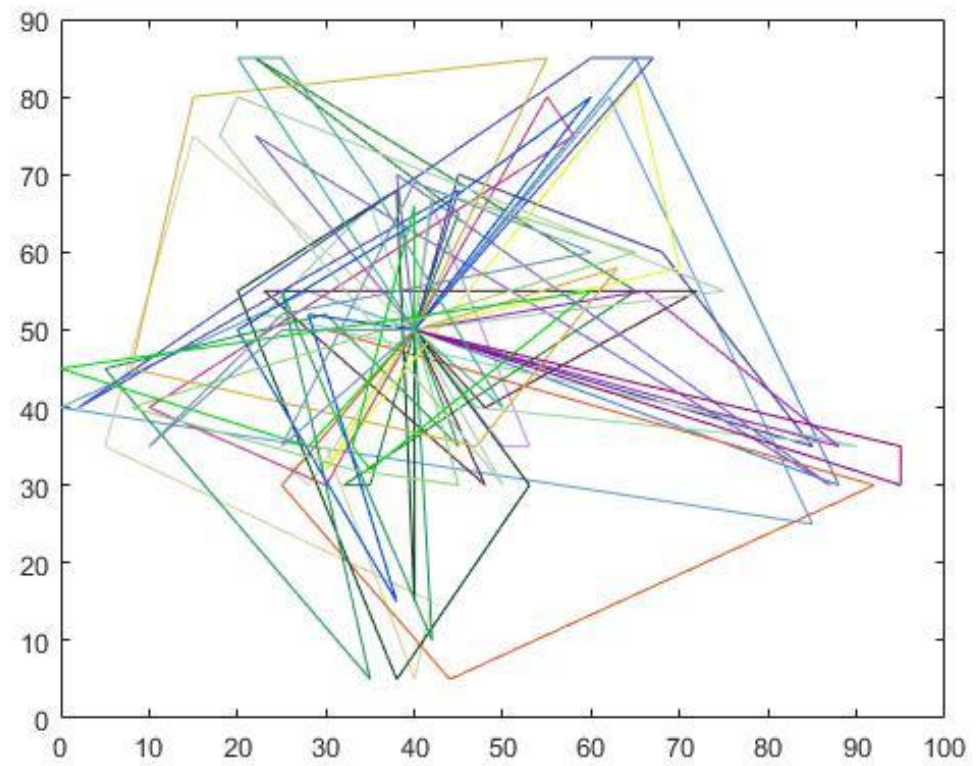

Figure 5. Distribution Routing of General Genetic Algorithm

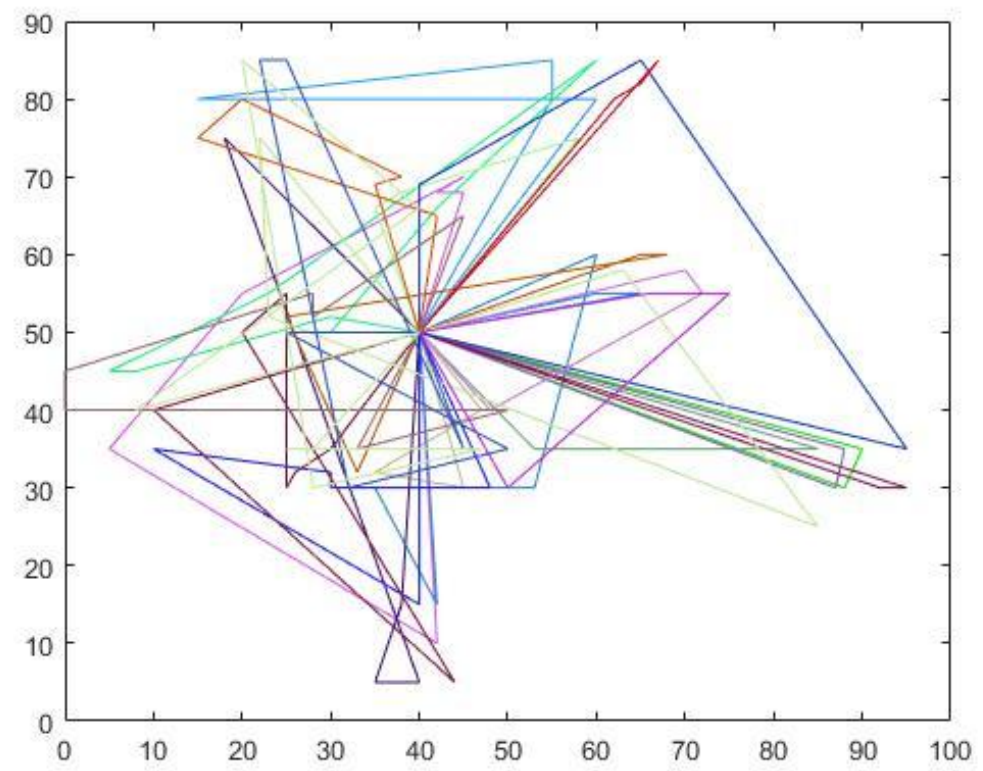

Figure 6. Distribution Routing after Adding in Hill Climbing Operation

\begin{tabular}{cccc}
\hline No. & Parameter & $\begin{array}{c}\text { Optimal } \\
\text { solution }\end{array}$ & $\begin{array}{c}\text { Reaching optimal solution } \\
\text { algebra }\end{array}$ \\
\hline 1 & $\begin{array}{c}\text { General genetic algorithm } \\
\text { Hybrid genetic algorithm adding with } \\
\text { hill climbing operation }\end{array}$ & 34385 & 891 \\
2 & 22952 & 624 \\
\hline
\end{tabular}

Table 3 Comparison among Optimal Experimental Solutions

\section{Conclusion}

The Thesis takes genetic algorithm as blueprint and is added with hill climbing operation, which comprehensively improves the performance of genetic algorithm, increases convergence speed and computational accuracy of genetic algorithm in solving cigarette distribution routing optimization, 
and prevents it from being sunk into local solution, thus greatly improving the efficiency of genetic algorithm.

\section{References}

[1] Savelsbergh M. local Search for Routing Problem with Time Window[R].Annals of Operations Research,1985,16(4):285-305.

[2] Baker J.E. Adaptive Selection Methods for Genetic Algorithms[D].In Proc. of the 1'Int'1.Conton Genetic Algorithms.1985:110-111.

[3] Olatz Arbelaitz, Clemente Rodriguez, Comparison of Systems Based on Evolutionary Search and Simulated Annealing to Solve the VRPTW Problem. International Journal of Computational Intelligence and Applications[J].2004,4(1):27-39.

[4] Jorg Homberger, Hermann Gehring. Two Evolution Metaheuristics for the Vehicle Routing Problem with Time Windows. INFOR[J].1999,37(3)

[5] Wee-Kit. A Hbrid Search Algorithm for the VRPTW. International Journal On Artificial Intelligence Tools[J].2001,10(3):431-339.

[6] B. Ombuki, M. Nakamura. M. Osamu. A Hybrid Search Based on Genetic Algorithm and Tabu Search for Vehicle Routing, presented at the $6^{\text {th }}$ International Conference on Artificial Intelligence and Soft Computing,Banff,Canada,.2002,9:176-181.

[7] Han Peng, Wang Quan. Development of Geographic Information System-Arc Engine Method, Wuhan: Wuhan University Press, 2008.

[8] Zhang Feizhou, Fan Yaozu, Traffic Control Engineering, Beijing: China Railway Publishing House, 2005.

[9] Yang Qing, Wei Zhongbang, A study on a logistics distribution system based on Arc GIS engine, Science of Surveying and Mapping, vol. 35, pp. 149-151, 2010.

[10] Wang Xiaowan, Luo Zhengshan, Li Zhou, Research of Vehicle Dispatch Based on GIS Logistic Distribution and Delivery, TONG JI YU JUE CE, vol. 2, pp. 54-56, 2011. 